\title{
CHANGES IN BLOOD PRESSURE IN BREAST CANCER SURVIVORS UNDER A PHYSICAL EXERCISE PROGRAM
}

José Jean de Oliveira Toscano', Kettury Maria da Silva Barros¹, Amaro Wellington da Silva', Naira Sabrina Ferreira da Costa', Vicente de Paulo Silva Moreira', Carlos Alencar Souza Alves Júnior², Diego Augusto dos Santos Silva²

${ }^{1}$ Universidade Federal de Alagoas - Maceió (AL), Brazil.

2Universidade Federal de Santa Catarina - Florianópolis (SC), Brazil.

Objective: The main aim of this study was to verify chronic and acute alterations in the systolic blood pressure (SBP) and diastolic blood pressure (DBP) in breast cancer survivors undergoing a physical exercise program. Methodology: In a reference hospital for cancer treatment in the city of Maceió, Alagoas, Brazil, a total of 24 female breast cancer survivors, 56.8 ( \pm 7.7 ) years old, underwent a physical exercise program. Their blood pressure was supervised through digital blood pressure monitors for the wrist, before and after the exercise sessions. To make comparisons in each session - pre- and post-exercise - the paired sample t-test analysis was applied. For the chronic effect analyses, the analysis of variance for repeated measures (ANOVA RM) was used to identify the possible differences in SBP and DBP variables, pre-exercise, throughout the 15 training sessions. A significance level of 5\% was considered. Results: Except for the fourth and sixth sessions, it was established that the SBP levels decreased in all sessions after they were completed ( $\mathrm{p} \leq 0.05)$. For DBP, there was a significant decrease only after the first three exercise sessions. Regarding chronic effects, there was a mean reduction in SBP values at rest, throughout the sessions, with a hypotensive effect above $70 \%$ from the seventh session on ( $\mathrm{p} \leq 0.05)$. Regarding DBP, differences in the DBP values at rest were enhanced from the tenth session onward, with a hypotensive effect above 94\%. By comparing the beginning of the program with the last session, a difference in SBP and DBP, of -9.0 and $-5.5 \mathrm{mmHg}$, respectively ( $\mathrm{p}<0.01$ ), was identified. Conclusion: Those survivors who joined the physical exercise program showed a chronic and acute decrease in both SBP and DBP levels.

Keywords: Cancer Survivors; Exercise; Arterial Pressure. 